\title{
Leakage Post Laparoscopic Sleeve Gastrectomy
}

\author{
Kamal Abdelrahman Abo Sonna ${ }^{1, *}$ MD, Hany Abdelfatah El-Hady ${ }^{1}$ MD, Hoda A. A. Yousef ${ }^{1}$ MD, \\ Zeinab Ahmed Ibrahim ${ }^{1}$ MD.
}

*Corresponding Author:

Zeinab Ahmed Ibrahim

zenabad49@gmail.com

Received for publication December 06, 2021; Accepted January 14, 2022; Published online January 14, 2022.

Copyright The Authors published by Al-Azhar University, Faculty of Medicine, Cairo, Egypt. Users have the right to read, download, copy, distribute, print, search, or link to the full texts of articles under the following conditions: Creative Commons Attribution-Share Alike 4.0 International Public License (CC BY-SA 4.0).

doi: $10.21608 /$ aimj.2022.109863.1712

${ }^{1}$ General Surgery Department, Faculty of Medicine (for girls), Al-Azhar University Cairo, Egypt.

\begin{abstract}
Background: Laparoscopic sleeve gastrectomy (LSG) is the most commonly performed bariatric surgery. Gastric leak is a known complication after LSG with reported incidence. Severe morbidity, including sepsis and multi-organ failure.

Aim of the work: To present patient who suffered from leakage after LSG and to review definitive surgical management and its outcomes.

Patients and methods: This study was conducted in department of general surgery at Al Zahraa university hospital from 2017 to 2019. 100 patients with morbid obesity underwent LSG among these all patients diagnosed with gastric leak were included other patients referred to our hospital with gastric leak were also included with analysis of the site of leak, time of presentation, investigation and treatment for all patients.

Results: Among 100 patients who underwent LSG, 6 cases (1.6\%) 3 patients from our hospital and the other 3 patients were referred from other hospital. The mean BMI was $45.7+5.5$ the mean age was 30 45years. Gastric leak was diagnosed 24 hrs. To 1 month with interval between surgery and readmission for leak was 13 days range (5-3odays) . We record one case death of 3 cases referred due to sepsis. Remaining 5 cases were fully recovered after surgical treatment, no sign of persist leak, no mortality during period of follow up.

Conclusion: Gastric leakage after LSG is associated with long-term morbidity and is one of the most serious complications and may end in organ failure and even death so choosing proper treatment should be done as early as possible and surgical repair with conversion to Roux-eny gastric bypass is very effective and safe.

Keywords: Laparoscopic sleeve gastrectomy; Gastric leak; Roux-en-y gastric bypass.
\end{abstract}

\begin{abstract}
Disclosure: The authors have no financial interest to declare in relation to the content of this article. The Article Processing Charge was paid for by the authors. Authorship: All authors have a substantial contribution to the article.
\end{abstract}

\section{INTRODUCTION}

Morbid obesity shows a growing epidemiology in western world and LSG is becoming the most popular for the treatment of morbid obesity and because of the permanent anatomical alternation following the bariatric operations, complications could occur any time during the course of a patient's life. ${ }^{1}$

For high-risk patients, LSG was recommended as the first step in a two-stage procedure aiming to reducing operative risk and comorbidities. ${ }^{2}$

Staple line leak one of the most common complications post LSG and this leak may be acute (early) or late (chronic) It occurs in up to $5 \%$ of patients following LSG ${ }^{1}$. Management depends on if there is intra-abdominal collection or no and the size of this collection. It also depends on extent of leak and the hemodynamic status of the patients. ${ }^{3}$ Gastric leakage (GL) post LSG has proven to be a disastrous complication leading to longer time of hospital stay and to use other modalities for rescue. ${ }^{4}$
GL is a catastrophic complication after bariatric surgery occurring in about $1-2 \%$ after LSG. This leak may be presented as peritonitis, abscess, fistula, sepsis and multi organ failure. ${ }^{5}$

In bariatric surgery the majority of post-operative death caused by staple line dehiscence and leak, so treatment of fistula and leak is critical especially after LSG as the staple line is long and the intraluminal pressure is elevated. ${ }^{6}$

Leak results in great health and economic burden to the patients and the health care facilities. ${ }^{7}$

Hemorrhage, narrowing of the tubular stomach, and gastric leakage are the most serious complications associated with stomach residue. A wide range of associated leak has been reported and ranged from $0.7-20 \%^{\cdot 6}$

Leakages that develop as a result of secondary ischemia appear 5 to 6 days after the operation. Leakages that develop due to technical issues are usually discovered within the first two days of the operation. Fever, epigastric pain, tachycardia, and 
leukocytosis are the most common symptoms in patients who develop leakages. $^{8}$

LSG leaks can present with a wide range of clinical symptoms, from mild left shoulder pain to severe sepsis and multiple organ failure, depending on the severity of the leak itself.

The most serious associated complication following LSG is the gastric fistula because this type of fistula is difficult to be closed as compared to other types of fistula and because of the lack of standardization in the management of the fistula, especially the chronic fistula. $^{9}$

It is necessary to have an increased level of suspicion in order to correctly identify a leak following an LSG. This treatment focuses on controlling sepsis and providing nutritional support. Each patient's care must be tailored to their specific needs. ${ }^{10}$.

\section{PATIENTS AND METHODS}

This is a retrospective study of patients who underwent LSG from 2017 to 2019 at Department of General Surgery at Al Zahraa University Hospital. 100 patients with morbid obesity, the mean BMI $45.6+5.5 \mathrm{~kg} / \mathrm{m} 2$ underwent LSG

Laparoscopic sleeve gastrectomy with Anti-embolic precautions were taken and appropriate preoperative antibiotics were administered. Under general anesthesia with endotracheal intubation, the patient was in supine position. A pneumoperitoneum was achieved using closed technique with Veress needle. Five ports were used; three ports of size $12 \mathrm{~mm}$ were placed as an arc at $15-18 \mathrm{~cm}$ below the xiphoid and 3 $\mathrm{cm}$ to the left of the midline (optical port) and right and left midclavicular lines (working ports). A 5-mm trocar port was placed along the left subcostal margin (assistant port) another 5-mm port was placed in the epigastric region for liver retraction. The operating table was placed in reverse Trendelenburg position and the left lobe of the liver was retracted cephalic to expose the esophageal hiatus. A $30^{\circ}$ angled laparoscope was used. Evacuation of gastric secretion and gas by nasogastric tube was done. The pylorus of the stomach was identified, and the greater curve of the stomach was elevated. Devascularization of the greater curvature of the stomach was done using the laparoscopic ultrasonic scalpel. A $35 \mathrm{Fr}$ orogastric bougie was placed adjacent to the pylorus. The dissection was started $5-8 \mathrm{~cm}$ from the pylorus and proceeds to the angle of His. A $60 \mathrm{~mm}$ black or green cartridge was used for stapling at the beginning (proximal to pylorus). The second cartridge, black or green, was applied proximal to the angularis with care taken to avoid stenosis here. Stapling continued until 1-2 cm gastroesophageal junction by using a golden cartridge. The resected stomach was grasped by a laparoscopic grasper through one of the working port sites. Testing for leakage by injection of methylene through the orogastric bougie was achieved. Drain was placed routinely.

Routine post-operative gastrograffin on the second postoperative day was done for all patients. Also Clinical symptoms or laboratory investigations suggesting leak after LSG as discomfort, pain and signs as abdominal distension, fever tachypnea, tachycardia, increased WBCs, CRP, all were recorded in all patients.

All patients with leak patients were evaluated by CT abdomen and pelvis and started broad-spectrum antibiotics. Patients were closely observed for any clinical deterioration with no oral intake Fig. (1)
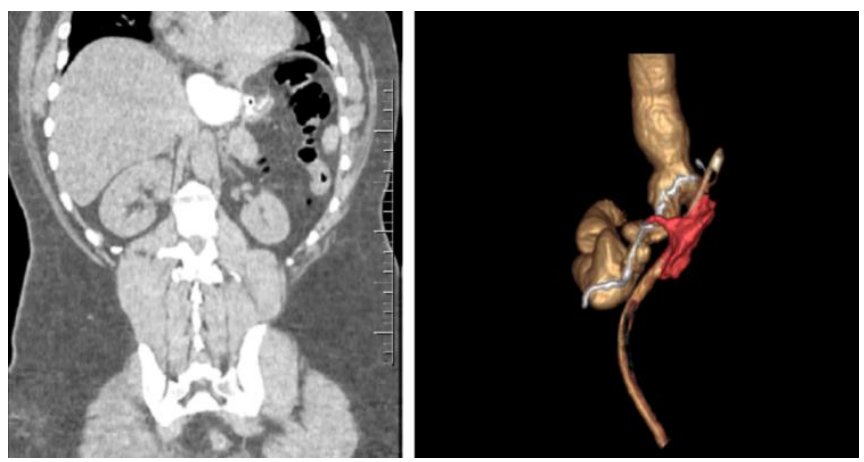

Fig. 1: Post sleeve CT scan with evidence of leak at the stable line in the middle portion

All data were collected demographic anthropometrics, comorbidities, operative and perioperative data, site of leak, time of presentation, method of detection and interval between detection and surgery.

Treatment of the patients with leak depends on hemodynamic state, presence of peritonitis, amount of collection and is the collection localized or diffuse. The decision between conservative and surgical exploration is made based on these circumstances.

In cases with suspected leaks abdomen and pelvis computed tomography with oral and intravenous contrast, gastrografin study, and upper endoscopy GI endoscopy. When there is localized collection or abscess in subphrenic space seen on CT it is treated by guided percutaneous drainage.

If the patient is hemodynamically stable and there is no generalized peritonitis gastrografin study with upper GI endoscopy is done to assess the size and the site of the leak and to assess if there is any stricture. If there is leak without stricture endoscopic stenting may be done. Follow up of body temperature, leukocyte count and drains output monitoring. If the patients' general condition improved, WBCs returned to normal, and the drain stopped draining, a CT scan was performed after one week to ensure there was no leak, and oral intake resumed. Patients were discharged after drain removal. Clinical examination and abdominal ultrasound are performed at 2 weeks, 4 weeks, and 12 weeks.

If the patient with leak is not stable we start with resuscitation and then laparoscopic exploration washing out and drainage. Feeding jejunostomy tube may be done at the same time. Postoperative observation of the amount and nature of drain output following surgery

According to Csendes et al., early, intermediate, and late leaks were classified as those that appeared 1 to 4,5 to 9 , and 10 or more days after surgery, respectively, after surgery. ${ }^{\mathbf{1 1}}$ 


\section{RESULTS}

100 patients were included in this study underwent for LSG 40 male and 60 females. The mean BMI was $45.7 \pm 5.5 \mathrm{~kg} / \mathrm{m} 2$ (Table 1 ).

The mean operative time was $90 \pm 15$ minutes range (60-15O minutes). The mean length of hospital stay was $4.5 \pm 0.5$ days range (10-12days) (Table 2). There were no major intraoperative complications or death was recorded.

There were 6 cases of leak recorded. There were 5 females and 1 male, their BMI were $46-50 \mathrm{~kg} / \mathrm{m} 2$ with mean operative time were 90 minutes range (70160minutes). The time of presentation between surgery and readmission were between (4 days -one month). We record 1 case acute (early leak) presented on the $4^{\text {th }}$ day after LSG, 3 cases presented (5-9days) intermediate and the 2 cases referred were presented by late leak after 10 days -1 month. Patients were clinically presented with variation between abdominal pain with collection, tachycardia, fever and septic shock (Table 3\&4).

One patient presented with delayed leak died from sepsis and respiratory distress. Diagnostic laparoscopy for the other case of delayed presentation with Inspection and irrigation of the abdomen. leak was confirmed with pneumatic test with conversion to Roux-en-y gastric bypass by creating 15-30 cc gastric pouch distal to the site of leak after that jejenum was transected with linear stapler at $40 \mathrm{~cm}$ after identification of ligament of treitz and the efferent limb was followed for $75 \mathrm{~cm}$ then jejunojejenstomy was done with linear stapler with putting wide pore drain tube near the site of leakage. The goal of drainage was placement of drains to create controlled fistula with use of antimicrobial agents, and nutrition consideration.

In one case Stent placement was used after IV sedation. Site of leak was found with endoscopic wire inserted into the stomach afterwards full covered stent with $240 \mathrm{~mm}$ length and $28 \mathrm{~mm}$ diameter with wire guide and finally stent is widely opened. CT guided percutaneous drainage and if no improvement after $48 \mathrm{hrs}$ of conservative treatment (Table 5).

\begin{tabular}{|cc|}
\hline Characteristics & Patients \\
\hline Male & $1(16.6)$ \\
Female & $5(83.3)$ \\
\hline BMI $(\mathbf{k g ~ m})$ & $48.510 .5($ range $37.6-77.2)$ \\
Age & $39.59 .9($ range $22-55)$ \\
\hline Hypertension & $3(50)$ \\
\hline Hyperlipidemia & $4(66.6)$ \\
\hline Diabetes mellitus & $3(50)$ \\
\hline Sleep apnea & $2(33.3)$ \\
\hline Time of hospital stay & 4.5 day (Range 10-12) \\
\hline
\end{tabular}

Table 1: Preoperative characters of the patients undergoing LSG.

\begin{tabular}{ccc}
\hline Cases number & First Symptom(s) & First Signs \\
\hline First & Abdominal pain & Fever, tachycardia \\
Second & Left flank pain & Fever, tachycardia, abdominal tenderness \\
Third & Abdominal pain & Fever, tachycardia, abdominal Tenderness \\
Fourth & Nausea & Fever, tachycardia \\
Fifth & Cough, vomiting & Fever, tachypnea, tachycardia \\
Sixth & Abdominal pain, nausea & Fever, tachycardia, abdominal tenderness \\
\hline
\end{tabular}

Table 3: Signs and symptoms of patients with leak after sleeve gastrectomy

\begin{tabular}{ccc}
\hline Case Presentation & Mean \pm SD (range) & $\begin{array}{c}\text { No. (\%) of patients with } \\
\text { gastric leak }(\mathbf{n}=6)\end{array}$ \\
\hline Abdominal pain & $6(100)$ \\
Fever & $38.5 \pm 0.7(37.7-40.0)$ & $6(100)$ \\
\hline Tachycardia (bpm; normal <80) & $117 \pm 8.2(110-129)$ & $6(100)$ \\
Tachypnea (breaths per minute; normal & 24 & $1(16.6)$ \\
Increased WBC count (normal <10.000 & $17.650 \pm 3.823$ & $4(66.6)$ \\
leukocytes /mm & $(14.000-22.300)$ & $3(50)$ \\
\hline Increased CRP (mg/I; normal <10) & $252 \pm 150(69-547)$ & \\
\hline
\end{tabular}

Table 4: Clinical presentation and laboratory investigations in patients with gastric leak

\begin{tabular}{llll}
\hline Cases & First Intervention & Second Intervention & Third Intervention \\
\hline
\end{tabular}




\begin{tabular}{cccc}
\hline First & Conservative & No & - \\
\hline Second & Conservative & Drainage under CT & - \\
\hline Third & Conservative & No & - \\
\hline Fourth & Conservative & Stent & Surgical laparotomy \\
\hline Fifth & - & Drainage under CT & Died \\
\hline Sixth & - & Surgical laparotomy & \\
\hline
\end{tabular}

Table 5: intervention in each case with leak.

\section{DISCUSSION}

Obesity is a global health issue that affects both adults and children. For this, in obese persons especially those with comorbidities, bariatric surgery, particularly LSG, is the most effective treatment method for maintaining weight loss. ${ }^{12}$

Many complications usually develop following LSG, with a great burden regarding the morbidity and mortality. The most reported serious complications are the hemorrhage and gastric leak and the latter is reported in $0.7-20 \%$ of the cases with 0.1 to $0.2 \%$ mortality rate. Early diagnosis and management of gastric leak after LSG are very difficult and still a matter of debate. ${ }^{12}$

During LSG, the reported gastric leak rate is (1.4$2.5 \%)$ and (16-20\%) for primary sleeve gastrectomy and reoperation respectively. The most common site for leak along the proximal third of stomach close to gastroesophageal junction due to increase intragastric pressure and impairment of peristaltic activity with occurring of ischemia. ${ }^{13}$

Gastric leak mostly occurs along the proximal third of the stomach, close to the gastroesophageal junction owing to high intragastric pressure in this area with decreased peristaltic activity and ischemia. $^{14}$

The incidence of leak in this study is $6 \%$ which is close to that found by $(7.5 \%)$. ${ }^{15}$ A study of ${ }^{16}$ reported that the rate of leak was (1-3\%) and 10\% in the primary surgery, and after reoperation, respectively. ${ }^{16}$

The multicenter study was carried out by ${ }^{17} 5400$ patients who had LSG were studied. It was reported that male gender had 2.5 higher relative risk for leak and BMI of $50-50.9 \mathrm{~kg} / \mathrm{m} 2$ had 1.6 higher risk. Smoking, an incorrect postoperative diet, corticosteroids, and immunosuppressive medication have all been associated with higher risk for both fistulas and leaks. ${ }^{18}$

Patients with leak may be totally a symptomatic who are diagnosed during routine postoperative imaging studies to signs and symptoms of a septic shock (fever, abdominal pain, peritonitis, leukocytosis, tachycardia, hypotension) (Table 4).

Anastomotic leak was detected in our study using a combination of clinical symptoms and indicators such as abdominal pain, nausea, vomiting, fever, tachycardia, and tachypnea. (Table 4) Tachycardia, fever, and abdominal pain are the most common, but are not specific to diagnose postoperative leak. ${ }^{19}$
Tachycardia was the first symptom in 7 of 214 individuals, according to Burgos et al (3.3 percent). Another study found that 9 out of 210 patients had gastric leaks. All cases of leak in our study had tachycardia, which is consistent to ${ }^{20}$ report of tachycardia in most cases of leak. ${ }^{21}$

The suspicion of postoperative leak is increased with presence of unexplained fever and tachycardia and this requires other confirmatory radiological investigations to confirm the diagnosis of leak. ${ }^{22}$

Patients with this triad of stomach discomfort, tachycardia, and fever should be treated as leaks unless proven otherwise, according to ${ }^{19}$

Fever is the most important clinical factor in diagnosing gastric leak post-sleeve gastrectomy according to Csendes et al and Dakwar et al.

This study showed fever was found in all 3 categories early, intermediate, and late. ${ }^{21}$, reported that fever was the most important clinical indicator of gastric leak. Also, he reported wide range of presenting symptoms from minimal left shoulder pain to symptoms of fever, abdominal tenderness, and multiple organ failure.

Tachycardia above 120 beats per minute (BPM) is a strong indicator of gastric leak and systemic compromise, according to other experts. ${ }^{16}$

In our study one case develop leak early and the remaining cases are diagnosed after discharge with good general condition and after doing oral gastrograffin as it is routinely done for all cases. Routine postoperative UGI has sensitivity and specificity of $5-10 \%$ and $100 \%$ respectively. ${ }^{7}$

The adoption of a categorization system for postsleeve gastric fistulas could be the first step in developing an algorithm or strategy for management. A 3 characters-based model was suggested by ${ }^{9}$ as follows: Time of appearance (early, intermediate and late); Location (proximal, mid or distal gastric); Severity (type I and II).

Although CBC, CRP are contributary factors in diagnosis of leak, they are not sensitive or specific. ${ }^{23}$

In this study two cases conservative treatment alone was sufficient to control leak. CT guided drainage was used to drain the collection. One of them needed surgical drainage later to control the collection. We used a stent in one case and it was successful to control the leak.

Because this tissue is compromised by a severe inflammatory disease, suturing the leak is not recommended. Instead, an exploratory laparoscopy is 
recommended to drain the area where the leakage has originated and to wash the infected fluid. ${ }^{24}$

If the tissue was in an early state of inflammation, immediate surgical intervention with washout drainage and suture was more effective than a more cautious approach in patients with early leaks. While a more conservative approach for intermediate and late leaks in clinically stable patients with adequate hydration, proton pump inhibitors, nil per OS, nutritional support, percutaneous drainage of any collection, and broad-spectrum antibiotic therapy, with weekly upper gastrointestinal series to ensure healing, more invasive approaches may be considered when there is any concern about healing. ${ }^{25}$

The poor tissues and inflammation are the main factors for failure of operative management of leak and the role of operation is limited to debridement and drainage as reported by Oshiro et al. ${ }^{26}$

Most patients who had their fistula sutured failed to seal it directly, either because the leak persisted (orifice could not be clearly detected) or because the suture failed (inflamed and friable tissue), especially after the third day after surgery. Because of this, to help the surgeon in identifying the defect and close it, it is suggested to use a simultaneous endoscopic intervention together with insertion of a guide wire (rendez-vous) from the stomach lumen. ${ }^{27}$

Esophagogastric stent and percutaneous drainage are used for unrepaired leakages (either with surgery or the treatment of retarded leakages after LSG). Oral feeding could be continued after placing the stent. ${ }^{10}$

Endoscopic implantation of self-expandable metal stents (SEMS) after bariatric surgery is a less invasive and successful option for repairing leakage with success rate of $88 \% .{ }^{28}$ Fig. (2)

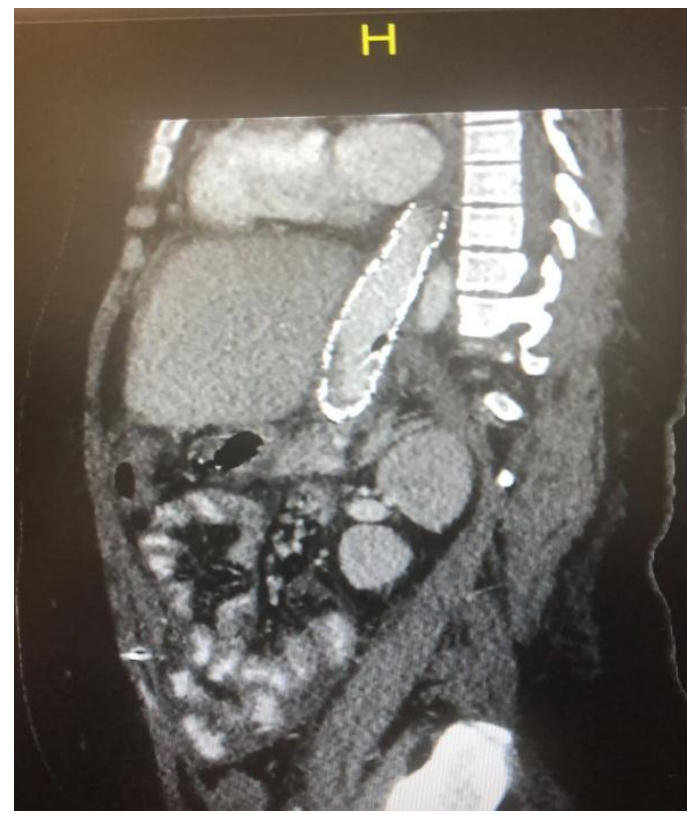

Fig. 2: Post self-expandable metal stent CT scan with evidence of no leakage

Also, according to Nguyen et al. and Serra et al. the use of a covered stent to control leak after sleeve gastrectomy was a safe and effective method with a success rate up to $83 \% .^{29}$

30, 31 included 29 patients who developed gastric leak after sleeve gastrectomy. The investigators used a stent to control the leak by putting the stents in situ for average 7 weeks duration. After the first stent was placed, 19 patients showed immediate success, whereas 5 patients required the implantation of a second stent. Two patients had continuous leakage that necessitated surgery.

In contrast, Tan et al. reported a 50\% success rate for gastric sleeve leak closure, with four patients requiring early stent removal due to migration, hematemesis, or obstruction from kinking at the proximal end of the stent. ${ }^{7}$

\section{CONCLUSION}

After LSG, abdominal pain, tachycardia, or fever should raise suspicions of a leak. The majority of patients had excessively high WBC and CRP levels. A strategy for dealing with leaks should be based on a case-by-case analysis. A valuable option is to utilize minimally intrusive strategies to handle leaks following LSG.

\section{REFERENCES}

1. Melissas J, Koukouraki S, Askoxylakis J, et al. Sleeve gastrectomy: a restrictive procedure?, Obes. Surg. 2007; 17: 57-62.

2. Lim R, Beekley A, Johnson DC, et al. Early and late complications of bariatric operation., Trauma Surg. Acute Care Open. 2018; 3: e000219.

3. Yehoshua RT, Eidelman LA, Stein M, et al. Laparoscopic sleeve gastrectomy--volume and pressure assessment., Obes. Surg. 2008; 18: 1083-8.

4. Casella G, Soricelli E, Rizzello M, et al. Nonsurgical treatment of staple line leaks after laparoscopic sleeve gastrectomy., Obes. Surg. 2009; 19: 821-6.

5. Papavramidis TS, Kotzampassi K, Kotidis E, et al. Endoscopic fibrin sealing of gastrocutaneous fistulas after sleeve gastrectomy and biliopancreatic diversion with duodenal switch., J. Gastroenterol. Hepatol. 2008; 23: 1802-5.

6. Erol V, Aydin C, Ugurlu L, et al. Treatment Methods for Leakage Occurring at Staple Line After Laparoscopic Sleeve Gastrectomy, J. Curr. Surgery. 2015; 5: 2-3.

7. Tan JT, Kariyawasam S, Wijeratne $\mathrm{T}$, et al. Diagnosis and management of gastric leaks after laparoscopic sleeve gastrectomy for morbid obesity., Obes. Surg. 2010; 20: 403-9.

8. Abraham A, Rizvon K, Singh J, et al. Successful Management of a Gastric Sleeve Leak with an Endoscopic Stent, Case Rep. Gastrointest. Med. 2012; 2012: 205979.

9. Csendes A, Braghetto I, León P, et al. Management of leaks after laparoscopic sleeve gastrectomy in patients with obesity., J. Gastrointest. Surg. Off. J. Soc. Surg. Aliment. Tract. 2010; 14: 1343-8. 
10. Warner DL and Sasse KC. Technical Details of Laparoscopic Sleeve Gastrectomy Leading to Lowered Leak Rate: Discussion of 1070 Consecutive Cases, Minim. Invasive Surg. 2017; 4367059.

11. Csendes A, Burdiles P, Burgos AM, et al. Conservative management of anastomotic leaks after 557 open gastric bypasses., Obes. Surg. 2005; 15 : $1252-1256$.

12. Donatelli G, Ferretti S, Vergeau BM, et al. Endoscopic Internal Drainage with Enteral Nutrition (EDEN) for Treatment of Leaks Following Sleeve Gastrectomy, Obes. Surg. 2014; 24: 1400-7.

13. Dakwar A, Assalia A, Khamaysi I, et al. Late complication of laparoscopic sleeve gastrectomy., Case Rep. Gastrointest. Med. 2013; 136153.

14. Ren CJ, Patterson E and Gagner M. Early results of laparoscopic biliopancreatic diversion with duodenal switch: a case series of 40 consecutive patients. Obes. Surg. 2000; 10: 514-23; discussion 524.

15. Kähler G. Anastomotic Leakage after Upper Gastrointestinal Surgery: Endoscopic Treatment, Visc. Med. 2017; 33: 202-6.

16. Abou Rached A, Basile M and El Masri H. Gastric leaks post sleeve gastrectomy: review of its prevention and management, World J. Gastroenterol. 2014; 20: 13904-10.

17. Benedix F, Benedix DD, Knoll C, et al. Are there risk factors that increase the rate of staple line leakage in patients undergoing primary sleeve gastrectomy for morbid obesity?, Obes. Surg. 2014; 24: $1610-6$.

18. Woźniewska P, Diemieszczyk I and Hady HR. Complications associated with laparoscopic sleeve gastrectomy-a review, Prz. Gastroenterol. 2021; 16: 5 .

19. Al Zoubi M, Khidir N and Bashah M. Challenges in the Diagnosis of Leak After Sleeve Gastrectomy: Clinical Presentation, Laboratory, and Radiological Findings., Obes. Surg. 2021; 31: 612-6.

20. Hamilton EC, Sims TL, Hamilton TT, et al. Clinical predictors of leak after laparoscopic Roux-en-Y gastric bypass for morbid obesity., Surg. Endosc. 2003; 17: 679-84.

21. Burgos AM, Braghetto I, Csendes A, et al. Gastric leak after laparoscopic-sleeve gastrectomy for obesity, Obes. Surg. 2009; 19: 1672-7.
22. Gagner M and Buchwald JN. Comparison of laparoscopic sleeve gastrectomy leak rates in four staple-line reinforcement options: A systematic review, Surg. Obes. Relat. Dis. 2014; 10: 713-23.

23. Björklund $P$, Lönroth $H$ and Fändriks L. Manometry of the Upper Gut Following Roux-en-Y Gastric Bypass Indicates That the Gastric Pouch and Roux Limb Act as a Common Cavity., Obes. Surg. 2015; 25: $1833-41$.

24. Szewczyk T, Janczak P, Janiak A, et al. Laparoscopic sleeve gastrectomy - 7 years of own experience., Wideochirurgia i Inne Tech. Maloinwazyjne = Videosurgery Other Miniinvasive Tech. 2014; 9: 427-35.

25. Rosenthal RJ. International sleeve gastrectomy expert panel consensus statement: Best practice guidelines based on experience of $>12,000$ cases, Surg. Obes. Relat. Dis. 2012; 8: 8-19.

26. Tamamori Y, Sakurai K, Kubo N, et al. Percutaneous transesophageal gastro-tubing for the management of anastomotic leakage after upper GI surgery: a report of two clinical cases, Surg. Case Reports. 2020; 6: 214.

27. Deitel M, Crosby RD and Gagner M. The First International Consensus Summit for Sleeve Gastrectomy (SG), New York City, October 25-27, 2007., Obes. Surg. 2008; 18: 487-96.

28. Tsai YN, Wang HP, Huang CK, et al. Endoluminal stenting for the management of leak following sleeve gastrectomy and loop duodenojejunal bypass with sleeve gastrectomy, Kaohsiung J. Med. Sci. 2018; 34: 43-8.

29. Serra C, Baltasar A, Andreo L, et al. Treatment of gastric leaks with coated self-expanding stents after sleeve gastrectomy. Obes. Surg. 207; 17: 866-72.

30. Nguyen NT and Wilson SE. Complications of antiobesity surgery., Nat. Clin. Pract. Gastroenterol. Hepatol. 2007; 4: 138-47.

31. Himpens Jacques, Dapri Giovanni, Cadière GuyBernard, Treatment of Leaks After Sleeve Gastrectomy: Bariatric Times, 2009. https://bariatrictimes.com/treatment-of-leaks-aftersleeve-gastrectomy/ (accessed November 9, 2021). 